POS PROCEEDINGS

\title{
A low-cost Cherenkov detector to be tested in CERN T9 beam line
}

\author{
Maria Rita Felici \\ Liceo Scientifico Temistocle Calzecchi Onesti, Fermo \\ E-mail: mariaritafelicielibero.it
}

TCO-4ASA (2016): Luca Cameli, Alessandro Camilli, Vanessa Castelli, Francesco Catalini, Samuele Cipolletti, Federico Crescenzi, Aurora De Paolis, Niccolò Decembrini, Francesco Fattenotte, Wafa Ghouzi, Francesco Lottatori, Benedetta Lucidi, Niccolò Minnucci, Giusi Valentina Pastanella, Michele Rastelli, Enrico Salerno, Alessia Vittori, Davide Zeppilli

Liceo Scientifico Temistocle Calzecchi Onesti, Fermo

TCO-ASA (2017): Roberta Barbieri, Davide Cartuccia, Luca Ciucci, Simone Giano, Letizia Manardi, Simone Pierantozzi, Marco Ricci, Alessandro Rongoni

Liceo Scientifico Temistocle Calzecchi Onesti, Fermo

\section{Evaristo Cisbani}

Istituto Superiore di Sanità e Istituto Nazione di Fisica Nucleare, Roma

E-mail: evaristo.cisbanieiss.infn.it

\section{Paolo Francavilla*}

Istituto Nazione di Fisica Nucleare, Pisa

E-mail: francavecern.ch

The TCO-Asa is a 2015 established team of high-school students of the Liceo Scientifico T. Calzecchi Onesti, in Fermo (Italy), who built a detector instrumented by consumer components including a CMOS camera and a silicon photomultiplier readout by the open-source ArduSiPM kit, to study the Cherenkov effect in water.

This proceeding presents the main activities of the students that included the definition and design of the detector geometry, the realisation of the external light-tight box by Scanny3D, the optimisation of the optical coupling between the water and the sensors, the experiences with ${ }^{18} \mathrm{~F}$ (prepared by Acom SRL) and ${ }^{226} \mathrm{Rd}$ radioactive sources, and the results of the tests performed at the Beam Test Facility in INFN-Frascati in 2016.

The team proposed to expose the detector to a test beam at CERN in 2016 and in 2017 within the Beamline for Schools initiative. The project was assessed among the best in 2016, and won the competition in 2017. A test beam with an improved detector has been performed in September 2017 at CERN, in the PS beam facility.

The European Physical Society Conference on High Energy Physics

5-12 July, 2017

Venice

\footnotetext{
*Speaker.
} 


\section{Introduction}

The activities of the TCO-Asa team started in 2015 in the Liceo Scientifico T. Calzecchi Onesti, in Fermo (Italy). The team is composed by a team leader, Maria Rita Felici, by almost thirty high-school students, and by two tutors (Evaristo Cisbani and Paolo Francavilla). The project got the support of local companies involved in three dimensional scanning systems and in radiopharmaceuticals production. The team designed and realised a low cost Cherenkov detector as an extracurricular didactic project. The project has three main purposes: learn how to design and build a detector, how to characterise it, and how to organise the work needed for the first two goals as a real experimental collaboration in high energy physics.

The interest for this extracurricular project has been triggered by the Beamline for Schools initiative, organized by CERN in Geneve. In the last four years, CERN gave access to a beam line (the T9 beam line) to project proposed by high-school students.

Among the several proposals by TCO-Asa team, the construction of a low cost Cherenkov detector has been selected for quite a few reasons:

- a conceptually simple detector that directly exploits an intriguing physics process: the Cherenkov effect which involves special relativity and quantum phenomena;

- it can be instrumented by consumer, commonly available, cost-affordable components;

- the detector can represent a sort of evolving laboratory of the school, used and improved by the student generations that follow one another.

The latter motivation played already a crucial role to give continuity to the project. In fact, the project was originally submitted to the Beamline for Schools initiative in 2016, resulting among the best proposals. An improved detector was proposed in 2017 by a renovated group of students (the first group was already in the last year of the high school). This new proposal won the competition and got beam time for tests that have been recently completed, and the analysis of the results will likely involve new students of the institute.

\section{The didactic project}

The proposed detector essentially consists of a water- and light-tight box filled with the liquid Cherenkov radiator; the box has one or more properly designed holes where consumer cameras can opportunely take pictures of the Cherenkov light produced by charged particles traveling water faster than light (e.g. electrons with kinetic energy above $260 \mathrm{keV}$ ).

Different detector geometries, optical properties of the box walls and sensor setups have been proposed and realized as part of an extracurricular project involving approximately 30 students of different ages from different classes. Each proposed box was built entirely by the students, supported by teacher and the personell of Scanny3D; they directly participated to the assembling of the mirrors (first box) or of the methacrylate plates (second and third box). Experiment was carried out using beta emitting radioactive sources placed in distilled water, taking into account all safety procedures required when handling radioactive sources. One of the radioactive substance $\left({ }^{18} \mathrm{~F}\right)$ was provided by Acom, a local company producing radioisotopes for medical applications; 
this also represented an interesting educational opportunity for the students. The analysis of the images recorded by various photographic instruments (Reflex camera, immersion camera, CMOS sensor) was carried out by all the students. In order to comprehend the experiment the students and their teacher were required to carry out an in-depth study of specific cross-sectional themes which are part of the ministerial programmes of study in high school Physics:

- Cherenkov effect: phenomenology and threshold effect and its applications [1,2].

- Propagation of optical light: reflection, diffusion, optical continuity, absorption

- Photo-sensors: photoelectric effect, semiconductors

- Digital image processing

- Radioisotopes and radioactive decays

To finalize the project, the students have been asked to prepare a video to present their project, and a web page to share with the rest of the school and outside the school, the status of the project, and the most recent findings. The web-page [4] collects didactic material on this project, a daily $\log$ of the activities, the full documentation of the projects, and several links to other initiative with similar topics.

\section{Experimental apparatus}

The main components of the experimental apparatus are the box, housing the Cherenkov radiator, the photo-sensors and the radioactive sources emitting beta particle, used to test the different detector configurations proposed.

In fact, the detector design has evolved and improved over the time taking into account the lessons learned during and from the tests performed by means of two radioactive sources emitting few $\mathrm{MeV}$ electrons which are above the Cherenkov threshold in water: a sealed, constant $15 \mu \mathrm{Ci}$ activity ${ }^{226} \mathrm{Ra}$ available in the school and fast decaying ${ }^{18} \mathrm{~F}$-FDG samples with activity selectable in the range $10 \mu \mathrm{Ci}$ to $700 \mu \mathrm{Ci}$.

Composition of the box, its size, the position of the cameras and the coupling of the photosensor to the box windows have been modified from the original design to improve Cherenkov light collection.

Distilled water has been chosen as Cherenkov radiator for its moderately large refractive index, good optical properties, easy handling with no relevant hazard and trivial procurement.

Commercial CMOS cameras and more suitable (but less popular) Silicon Photomultiplier (SiPM) have been considered as photo-sensors; the dark noise, strongly correlated to sensor temperature has been immediately identified as one of the major issue to be mitigated: camera (ASI174) with temperature probe and integrated thermoelectric cooling to few degree Celsius has been adopted.

The final setup of the detector, also used in the recent test beam at CERN, essentially consists of (see figure 1): 


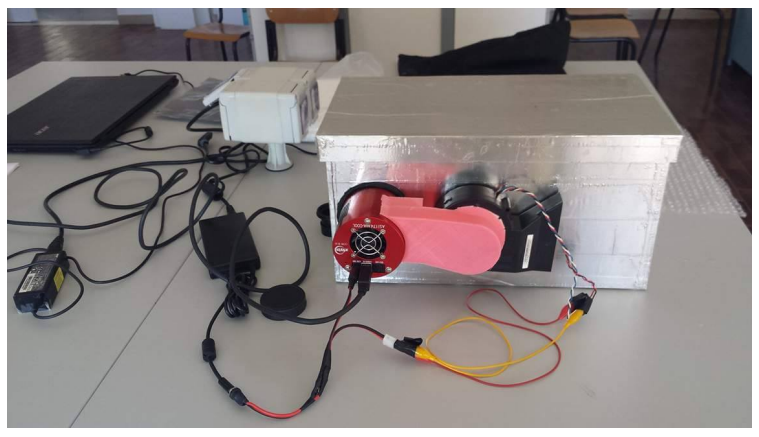

Figure 1: Final setup of the Cherenkov detector.

- Box: $20 \mathrm{~cm} \times 20 \mathrm{~cm} \times 40 \mathrm{~cm}$, made of polymethyl methacrylate and internal walls coated by Tywek upholstery.

- Photo-Sensors:

- CMOS ASI174 cameras designed to have very low power consumption (300mA at 5V). Thermo-electric cooling to reduce dark current noise for long exposures.

- SiPM from the ArduSIPM project [3], including an Hamamatsu MPPC for precision measurement, photosensitive area $1.3 \times 1.3 \mathrm{~mm}$.

\section{Results}

An important part of the project as been devoted to the data preparation, preservation and analysis. The team collected several thousand of photos, and the development of a system to log the experimental conditions has been fundamental to extract information from the photos.

In the first step of the experiment, this has been achieved by using a tag for each photo, and by taking note of all the photos and tags. In preparation for the experiment at the T9 beam line, a logbook system, a shared spreadsheet, and the use of run numbers to identify the conditions has been adopted.

The analysis of the data has been done in two frameworks. The first is based on ROOT; the second on Mathlab. The first framework consists of a first step in which the photos are transformed in ROOT's ntuples, and a second step in which the ntuples are analysed. The Mathlab based analysis is done by a monolithic code completely developed by the students. The analysis is based on the characteristic of the spectrum of the ADC counts in each pixel of a certain photo, and on statistics based on this spectrum. Among the others, the integral of the spectrum (the brightness of the photo) and the tails of the spectrum have been studied in details.

To characterise the sensor, several photos have been taken to study the noise as a function of the temperature, the exposition time, the gain of the pixels. In addition, an extensive study has been done to discover (and fix) potential leaks of the box tightness which would allow external light to enter. 
Figure 2 shows the dependence of the difference of the brightness between photos with the ${ }^{226} \mathrm{Ra}$ source inside the box and the photos without the radioactive source, normalized by the exposition time, as a function of the exposition time. In presence of the Cherenkov radiation, we expect the difference to be proportional to the amount of collected light, which is proportional to the exposition time. Some mild excess of brightness in the photos with the radioactive source is seen, but it is not sufficient to establish the nature of this signal. While no clear evidence of the Cherenkov

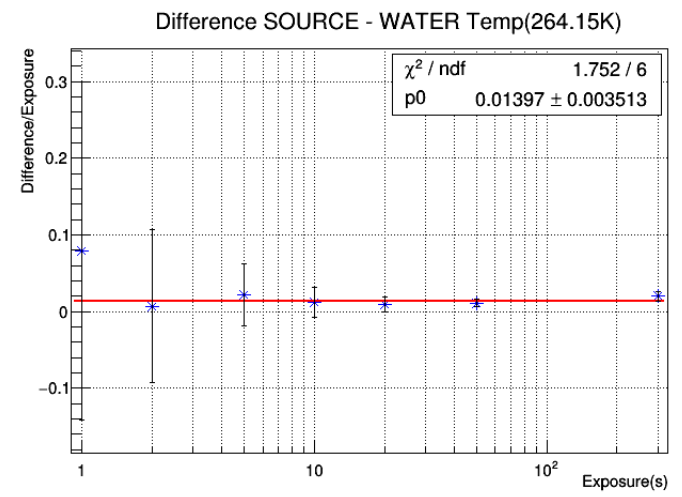

Figure 2: Dependence of the difference of the brightness between photos with the 226Ra source inside the box and the photos without the radioactive source, normalized by the exposition time, as a function of the exposition time.

light has been recorded when using the radioactive sources, very positive preliminary results have been obtained at with the T9 beam line. These results will be reported in future publications.

\section{Conclusion}

The TCO-Asa team, composed by high-school students, worked in the last three years to build a low-cost Cherenkov detector to be tested in the T9 beamline at CERN, thanks to the Beamline for School initiative. The students worked as in a small HEP collaboration to build, test and report their results: different configurations have been proposed, realized and tested helping improving the detector development and getting new students involved. The existing detector and the collected data will serve for and be used by next generations of students of the high-school T. Calzecchi Onesti in Fermo.

Authors warmly thank Acom and Scanny3D staffs and in particular Ing. Tonino Ciucchi, and Dr. Valerio Bocci of INFN Rome, for their precious support during the project.

\section{References}

[1] E. P. Cherenkova, The discovery of the Cherenkov radiation, Nuclear instruments and Methods A 595 (2008) 8-11

[2] L.D. Landau et al. Electrodynamics of Continuous Media, 1981

[3] V. Bocci et al. The ArduSiPM a compact transportable Software/Hardware Data Acquisition system for SiPM detector, Nuclear Science Symposium and Medical Imaging ConferenceâẮ́, IEEE, 2014.

[4] Web Page of the project: http://www.tco-beamline.com/ 